\title{
An Analysis of SARS-CoV-2 on the Molecular and Subatomic Levels through Applied I-Theory
}

\author{
H. H. Swami Isa, Christophe Dumas \\ Global Energy Parliament, Trivandrum, India \\ Email: isa@global-energy-parliament.net,dumas@global-energy-parliament.net
}

How to cite this paper: Isa, H.H.S. and Dumas, C. (2021) An Analysis of SARS-CoV-2 on the Molecular and Subatomic Levels through Applied I-Theory. Advances in Microbiology, 11, 75-89.

https://doi.org/10.4236/aim.2021.112006

Received: December 30, 2020

Accepted: February 17, 2021

Published: February 20, 2021

Copyright $\odot 2021$ by author(s) and Scientific Research Publishing Inc. This work is licensed under the Creative Commons Attribution International License (CC BY 4.0).

http://creativecommons.org/licenses/by/4.0/ (c) (i) Open Access

\begin{abstract}
The novel SARS-CoV-2 virus is spreading very effectively and is highly contagious. The SARS-CoV-2 virus is not acting like other viruses and many various symptoms in the human body have been observed. The analysis of the SARS-CoV-2 structure in light of the functioning of the CRISPR-Cas9, Cas12a and CasX mechanism suggests that the virus is man-made through genetic modification. Vaccination currently appears to be a feasible and promising means of pandemic containment. The first vaccines have been approved even though the mechanism of action of the virus is not yet fully understood. Two of the prominent vaccines are mRNA vaccines, which use genetic engineering. It cannot be ruled out that the mRNA vaccines would negatively affect human cells as well. This paper provides an analysis of the SARS-CoV-2 virus mechanism, based on an emerging unified quantum theory called the I-Theory, which gives a deeper understanding of the concepts of entropy and negentropy in the field of health. The analyses are developed on the molecular and subatomic levels in order to demonstrate how the entropy-negentropy balance is disrupted and the effects upon infected cells. It is shown that the base and the ionized oxygen atom of the RNA phosphate group have been replaced by a hydroxyl radical. On a subatomic level, this modification results in one electron (a fermion) missing from the phosphate base. It is assumed that due to the missing fermions and resulting higher levels of entropy, SARS-CoV-2 has a very high level of repulsion force, destroying the cytoplasm. The entropic effect leads to a sort of ballooning and weakening of infected cells, and in the case of low immunity or diseased cells, creates damage to the organs and various long-term effects.
\end{abstract}

\section{Keywords}

SARS-CoV-2, COVID-19, mRNA, Genetic Engineering, CRISPR, Boson, Fermion, Entropy, Negentropy, I-Theory 


\section{Introduction}

At the beginning of 2020, no one could have imagined the profound worldwide impacts that the Corona pandemic would have upon public health, society and the economy. The pandemic is caused by the novel SARS-CoV-2 virus, which appears to be spreading very effectively and is highly contagious. SARS-CoV-2 does not act like other viruses, and many diverse symptoms have been observed. By the middle of December 2020, 78 million people were infected and 1,700,000 people had died. In 2020, scientists around the world were focused on understanding how the virus works, as well as finding an effective vaccine to contain it. In December 2020, the first mRNA vaccines were approved worldwide, in an unprecedented timeline for development and approval. However, the mechanism of action of the virus is not yet fully understood, and it may also negatively affect the efficacy of the vaccines and/or create long-term health consequences that have not yet been observed.

In this article, the authors analyze the mechanism of the SARS-CoV-2 virus based on an emerging unified quantum theory called the I-Theory [1]. The theoretical context provides a deeper understanding of the concepts of entropy and negentropy [2] in the field of health and, more urgently, the mechanism of action of the SARS-CoV-2 virus. The analyses are developed on the molecular and subatomic levels in order to demonstrate how the entropy-negentropy balance is disrupted and the resulting effects upon infected cells.

In the first section, the authors illustrate that it cannot be ruled out that the virus is manmade through genetic modification. This assumption is substantiated by current literature on the CRISPR-Cas9, Cas12a and CasX mechanism. The genome analysis of SARS-CoV-2 shows a suspected insertion between the S1 and S2 proteins. In nature, there is no other example of a Coronavirus where the S1 and S2 proteins are not contiguous. Moreover, a study by M. Seyran and al shows that these insertions are not due to natural recombination [3]. According to T. Peacock et al., this specific insertion between S1/S2 plays a key role for transmission [4].

In the second section, the authors conduct an analysis of the results of this insertion on the molecular level. It is determined to be stable on the molecular level.

The third section presents a new area of research to better understand viruses and their genetic makeup, using a subatomic analysis. The result of the genome modification to SARS-CoV-2 creates major instability on the subatomic plane, even while it is stable at the molecular level. Being a more fundamental plane of energy, the potential for destruction is much higher, due to increased levels of entropy.

This article gives a new perspective to the functioning of the SARS-CoV-2 virus in the efforts to combat or find a treatment for the virus. It also raises some questions about the safety of mRNA vaccines. 


\section{SARS-CoV-2 Virus on the Molecular Level}

In this section we will discuss the possibility of a modified genome virus and the available tools for such a modification. We also discuss the consequences of modification on the molecular level.

\subsection{Review of the CRISPR-Cas9 Mechanism}

In 1993, the CRISPR (Clustered Regularly Interspaced Short Palindromic Repeats) system was discovered in prokaryotes (bacteria and archaea). Many of them have short DNA sequences in their genome that are regularly repeated along their genome [5]. In 2007, researchers concluded that CRISPR's function is related to prokaryotic immunity [6]. Following a virus infection, DNA sequences of the virus are integrated within these CRISPR sequences. Upon re-infection by this virus, the viral DNA within the CRISPRs is copied into RNA and is associated with the Cas9 protein (for CRISPR-associated protein 9). This Cas9-associated RNA binds to the DNA of the virus and Cas9 inactivates the viral DNA by cutting it. The virus is thus eliminated. CRISPR/Cas9 allows bacteria to retain the memory of a virus infection to better defend itself the next time they are infected.

It took the combined efforts of several research groups over the next 5 years to elucidate the underlying molecular mechanism behind the CRISPR system. In 2012 Emmanuelle Charpentier and Jennifer Doudna succeeded in hijacking this biological system to turn it into a tool capable of targeting and reshape specific locations in the genome of any cell [7].

The CRISPR system comprises two components: a single guide RNA (sgRNA) that is specific to the target DNA sequence and a CRISPR-associated endonuclease (Cas) (Figure 1). The Cas protein functions as a pair of molecular scissors, while the gRNA is the GPS that guides it to the appropriate site. In prokaryotes, the sgRNA guides the nuclease to viral DNA, but as a biotechnological tool, the design specifications of the sgRNA can be altered to target any organism's genome at virtually any location.

\subsection{Inhibition of the Genetic Sequence in DNA}

Nowadays, the CRISPR/Cas9 method is commonly used in genetic engineering. For many CRISPR-Cas experiments, Cas9 facilitates the gene editing process by creating a Double Strand Break (DSB) in the DNA (Figure 2). Generating a DSB is the initial step in the CRISPR editing pathway. The repair mechanism that follows will dictate the type of gene editing that occurs and will influence the desired editing outcome.

There are two main types of repair: non-homologous end joining (NHEJ), (if the number of nucleotides inserted or deleted is not divisible by three), and homology-directed repair (HDR) (if the number of nucleotides inserted or deleted 


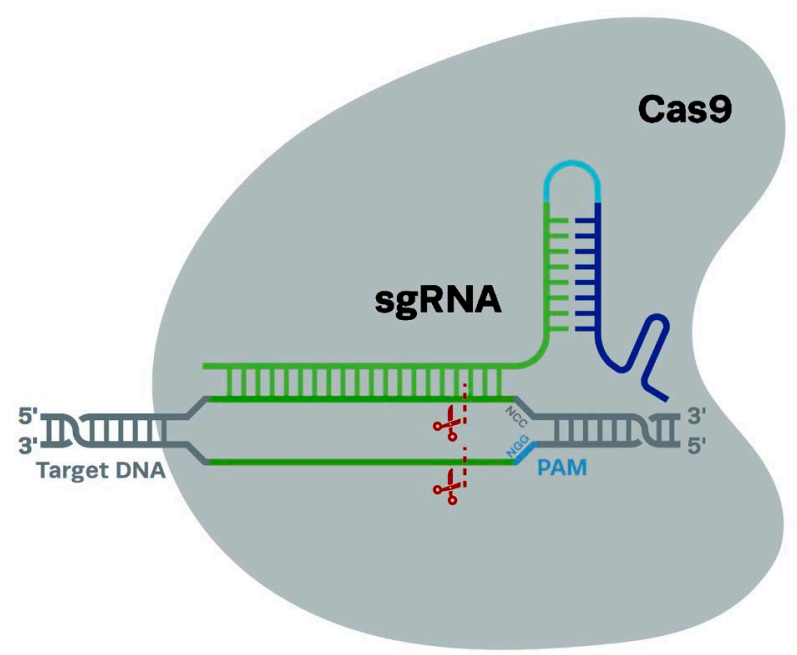

Figure 1. CRISPR-Cas9 system [8].
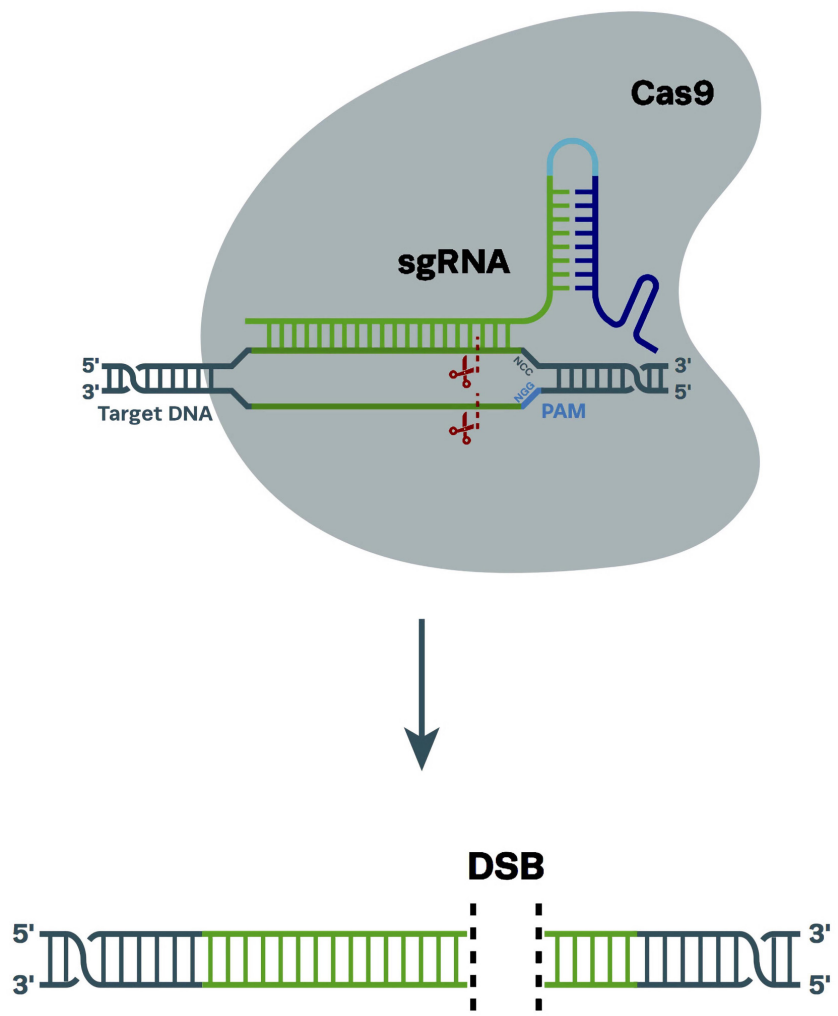

Figure 2. CRISPR-Cas9 generating a DBS in DNA [8].

is divisible by three) [8]. NHEJ results in either insertions or deletions of nucleotides to repair the DSB. HDR repairs the DSB by incorporating a DNA donor template into the target sequence (Figure 3 ).

\subsection{Cas12a and CasX-The Most Powerful CRISPR Mechanisms}

Cas9 is not the only protein that can be used in genome engineering. By using CRISPR-Cas9 researchers cannot be sure that they are only cutting a very precise 


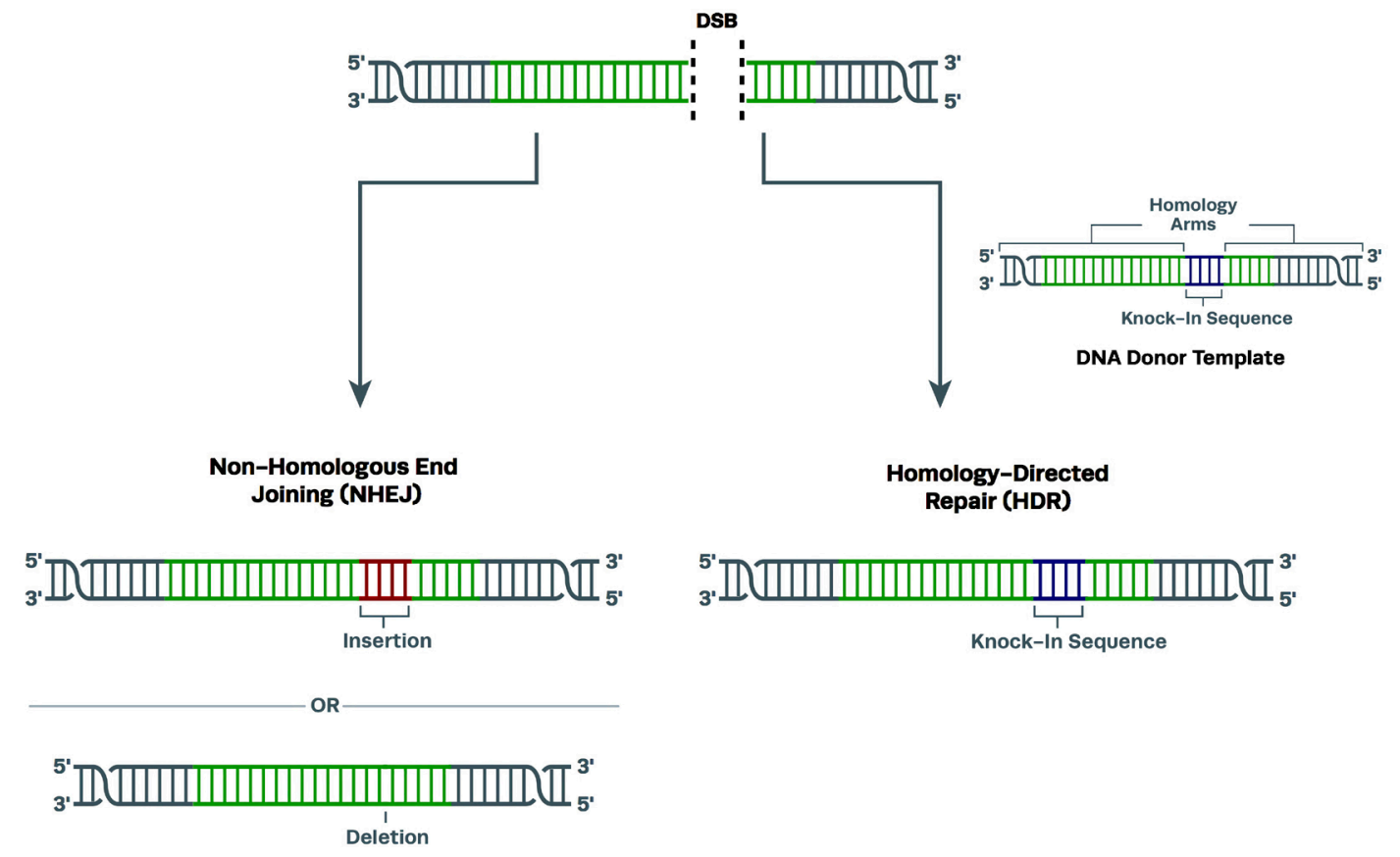

Figure 3. CRISPR-Cas9 as insertion, deletion or Knock-in DNA sequence [8].

portion of the genome. The CRISPR-Cas12a is more precise and specific. Cas9 only recognizes 2 or 3 nucleotides to bind firmly to DNA, while Cas12a "acts more like a velcro, by multiplying the weak bonds. All the nucleotides in the genetic sequence must be recognized for solid binding to take place" [9]. Cas12a lacked an inhibitor or modulator to allow its general use. Recent studies have shown that molecules AcrVA1 and AcrVA4 are able to block or modulate the enzymatic activity of CRISPR-Cas12a [10]. This discovery enables CRISPR-Cas12a to be used more safely. CRISPR-Cas12a offers scientists the possibility to easily cut a precise portion of the genome, opening the way to a multitude of applications ranging from the creation of genetically modified mice to the correction of genetic diseases.

Even if Cas9 and Cas12a have the same functionalities, they evolved independently [11] and use distinct mechanisms of DNA cleavage. Cas9 uses two active sites to generate a blunt DNA double-strand break near the PAM while Cas12a uses a single active site to make staggered cuts distant from the PAM, and the same active site can cleave free single-stranded DNA [7] [12]. The enzyme has been activated by specific target binding, this active site is non-specific (Figure 4). Recent studies on this enzyme show that Cas12 initiates DNA cutting by forming a 20-nucleotide R-loop in which the guide RNA displaces one strand of a double-helical DNA substrate. Until now, crystal structures and biochemical data have not explained how the second strand is cut to complete the double strand break. Recently, Cofsky et al. detected intrinsic instability in DNA flanking the RNA-3' side of R-loops, which Cas12a can exploit to expose second-strand DNA for cutting. Interestingly, DNA flanking the RNA-5' side of R-loops is not intrinsically unstable. This asymmetry in R-loop structure may explain the 
(a)

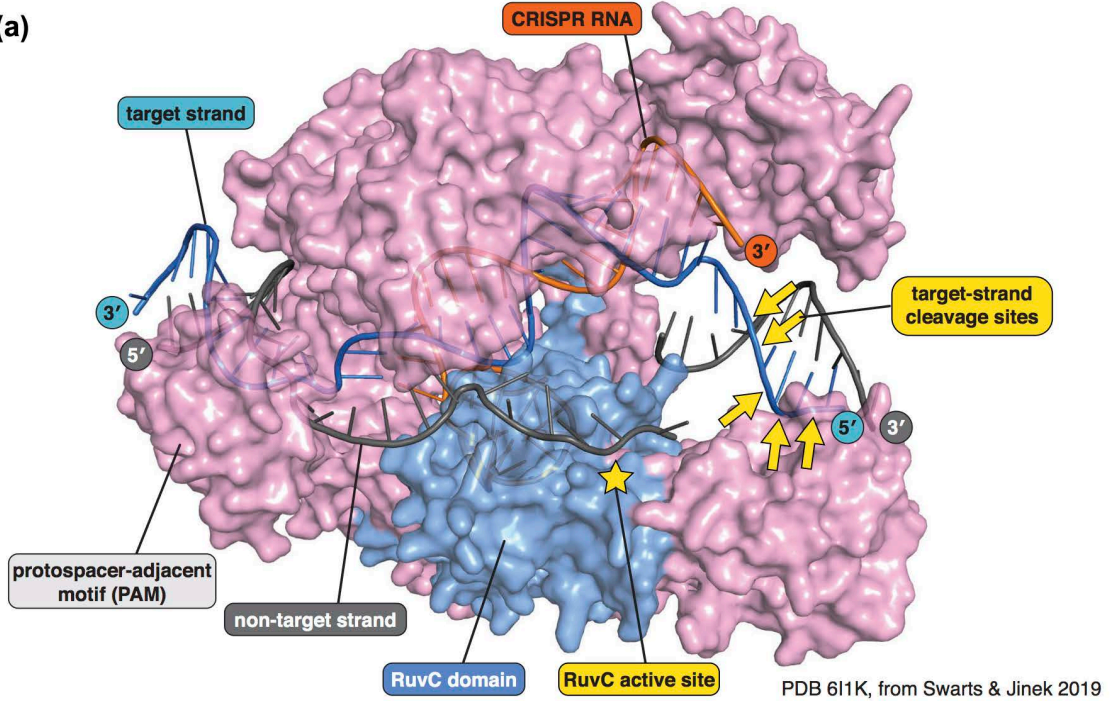

(b)
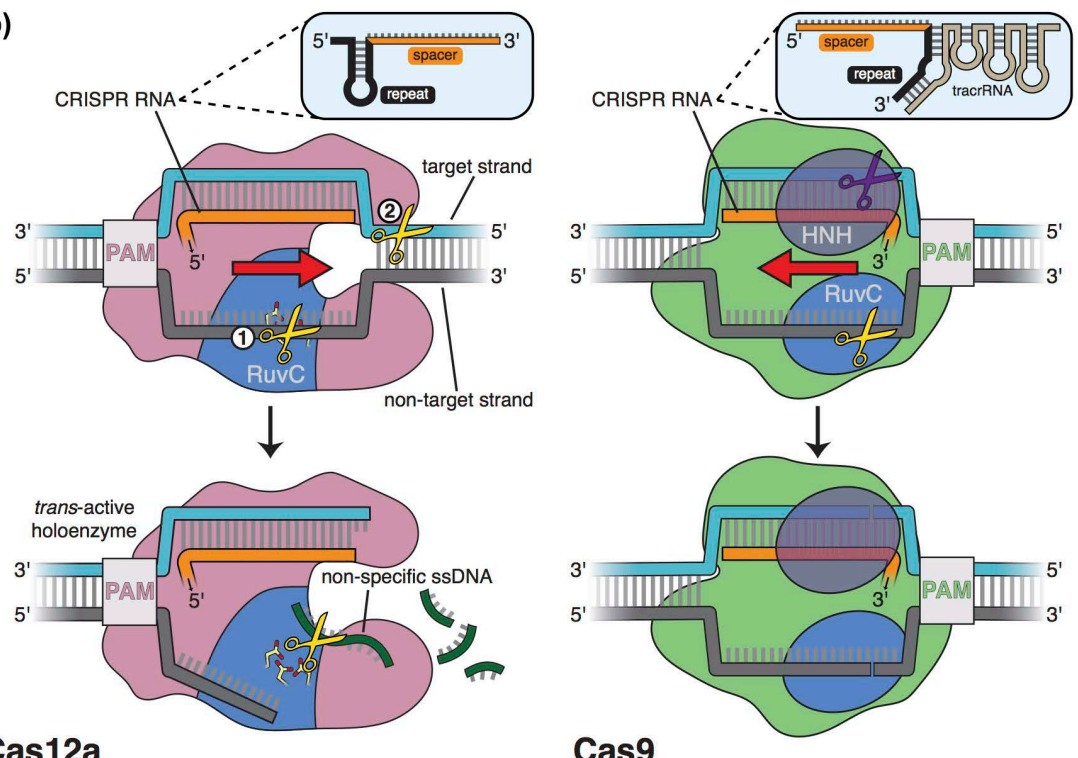

Figure 4. DNA cleavage mechanisms used by Cas12a and Cas9 [13].

uniformity of guide RNA architecture and the single-active-site cleavage mechanism that are fundamental features of all type V CRISPR-Cas systems [13].

Additionally, the position of the 20-nucleotide spacer sequence in Cas12a is opposite to that in Cas9 3'end versus 5' end, respectively, and consequently, R-loop formation by each enzyme family occurs with opposing directionality (Figure 4(b)).

In 2019, Jill Banfield and Jennifer Doudna, founded a third category of protein called CasX. This protein is smaller than Cas9 and therefore CasX is able to enter more easily inside a cell. Moreover, coming from non-pathogenic bacteria, CasX is more easily accepted by the human immune system [14].

CasX is a powerful and efficient gene editor in bacteria and human cells. Similar in structure to Cas9 or Cas12a, it has however evolved independently of oth- 
er Cas proteins. It can cut double-stranded DNA like Cas9 and Cas12a. CasX can bind to DNA to regulate genes and can be targeted to specific DNA sequences like other Cas proteins.

Cryo-electron microscopy revealed that CasX has an unanticipated quaternary structure in which the RNA scaffold dominates the architecture and organization of the enzyme (Figure 5). A RNA-guided DNA endonuclease generates a staggered double-stranded break in DNA at sequences complementary to a 20-nucleotide segment of its guide RNA [15] CasX complex contains also a full R-loop (45 bp DNA substrate) and ternary complexes.

\subsection{Is SARS-CoV-2 a Natural Virus?}

SARS-CoV-2 is a very unusual virus. It is extremely contagious, and recently discovered mutations are up to $70 \%$ more contagious than the original [16]. SARS-CoV-2 can travel through air and water. It can bind itself to dust particles in air and stay active for $6-8$ hours. The virus can also stay active on stainless steel surfaces for 6 days. The multiplicity of the symptoms in patients is almost unprecedented. Fever, cough and lung infection, strong headache, loss of smell and taste, renal injury, blood disease, kidney dysfunction, heart injury, loss of hair, loss of memory, extreme tiredness, and hypoxia are some of the common symptoms. Many recovered patients still have after-effects for a long period (including breathing difficulties, tiredness and loss of memory). It is reported that $60 \%$ of cured people still have heart problems [17]. After some weeks, some patients stop producing antibodies and a few has been infected twice.

Since genome editing allows human beings to easily modify DNA or RNA, many scientists wonder if SARS-CoV-2 is a natural virus. While many scientists claim that SARS-CoV-2 virus is a natural virus originating from a bat or pangolin, some studies claim the opposite. One study notes that since SARS-CoV-2 is $96 \%$ similar to the bat coronavirus RaTG13, there is strong support for the belief that

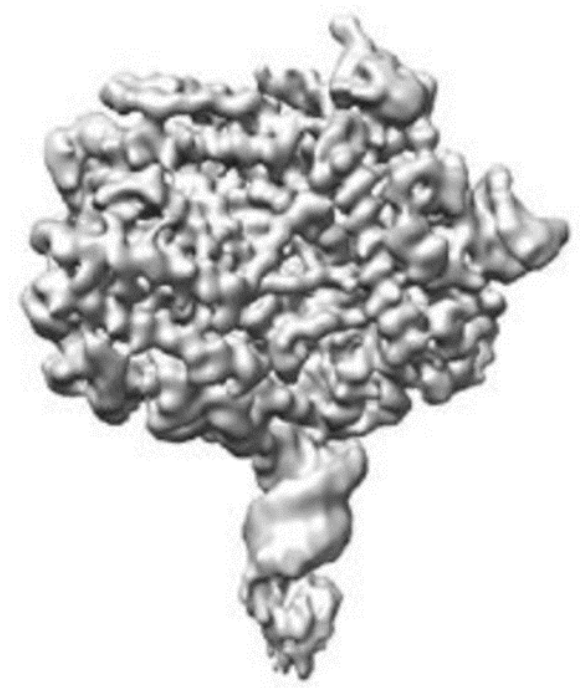

Figure 5. Structure of CasX [15]. 
the SARS-CoV-2 is a natural mutation of it. However, the flat and non-sunken surface of SARS-CoV-2's spike protein (S protein) conflicts with the general adaptation and survival patterns observed for all other natural coronaviruses. It seems likely there has been a recombination between the S1/S2 domains of S protein. The SARS-CoV-2 spike protein does not have any apparent further recombination, which conflicts with the usual recombination models of other coronaviruses. Similarly, the $\mathrm{S}$ protein receptor-binding domain of SARS-CoV-2 has not accumulated high-frequency non-synonymous substitutions [3]. A second article explains that these unique configurations of the spike protein are playing a key role for transmission due to enhanced replication in airway cells [4].

Finally, a third article expressed the same doubts about the fact that SARS-CoV-2 is a natural virus. Sørensen et al. showed that SARS-CoV-2 contains inserted sections in between S1/S2 [18] (Figure 6 and Figure 7). In nature, all coronaviruses have two contiguous spike proteins ( $\mathrm{S} 1$ and $\mathrm{S} 2$ ), therefore SARS-Cov-2 is exception. Moreover, they pointed out that SARS-CoV-2 uses not only the usual ACE2 receptor for binding the virus to the cell, but also a salt bridge. Sørensen states that the main method of binding is specifically related to cumulative charge from inserted sections placed on the SARS-CoV-2 Spike surface. These charges create an efficient salt bridge which binds the SARS-CoV-2 to the cell [18]. Sørensen has shown the presence of a high concentration of positive charged surface exposed amino acids within the receptor domain next to the receptor binding motif.

This study is also confirmed by the renowned Australian vaccine scientist Piplani has shown that SARS-CoV-2 has the highest energy binding to human cells as opposed to animal cells [20]. He expected to find an animal that was more susceptible to it, but he did not succeed. Piplani said: "It is almost perfectly human adapted, it couldn't do any better. We have to ask how that happened. Was it a complete fluke? It can be that nature has many shots at goal and you only see the ones that land. Another possibility which still cannot be excluded is

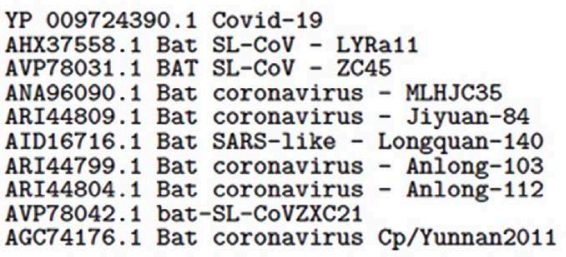

\begin{tabular}{|c|}
\hline $\begin{array}{l}72 \\
\text { GTNGTKR }\end{array}$ \\
\hline $\begin{array}{l}\text { NAATKR } \\
\text { SDRQVY } \\
\text { SDKIVY } \\
\text { SDRYTY. }\end{array}$ \\
\hline \\
\hline $\begin{array}{l}\text { NAATKR } \\
\text { SDRQVY. }\end{array}$ \\
\hline
\end{tabular}

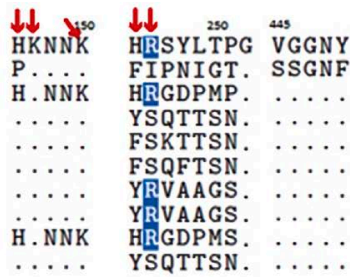

Figure 6. Alignments of corona virus spike protein inserts [18]. 


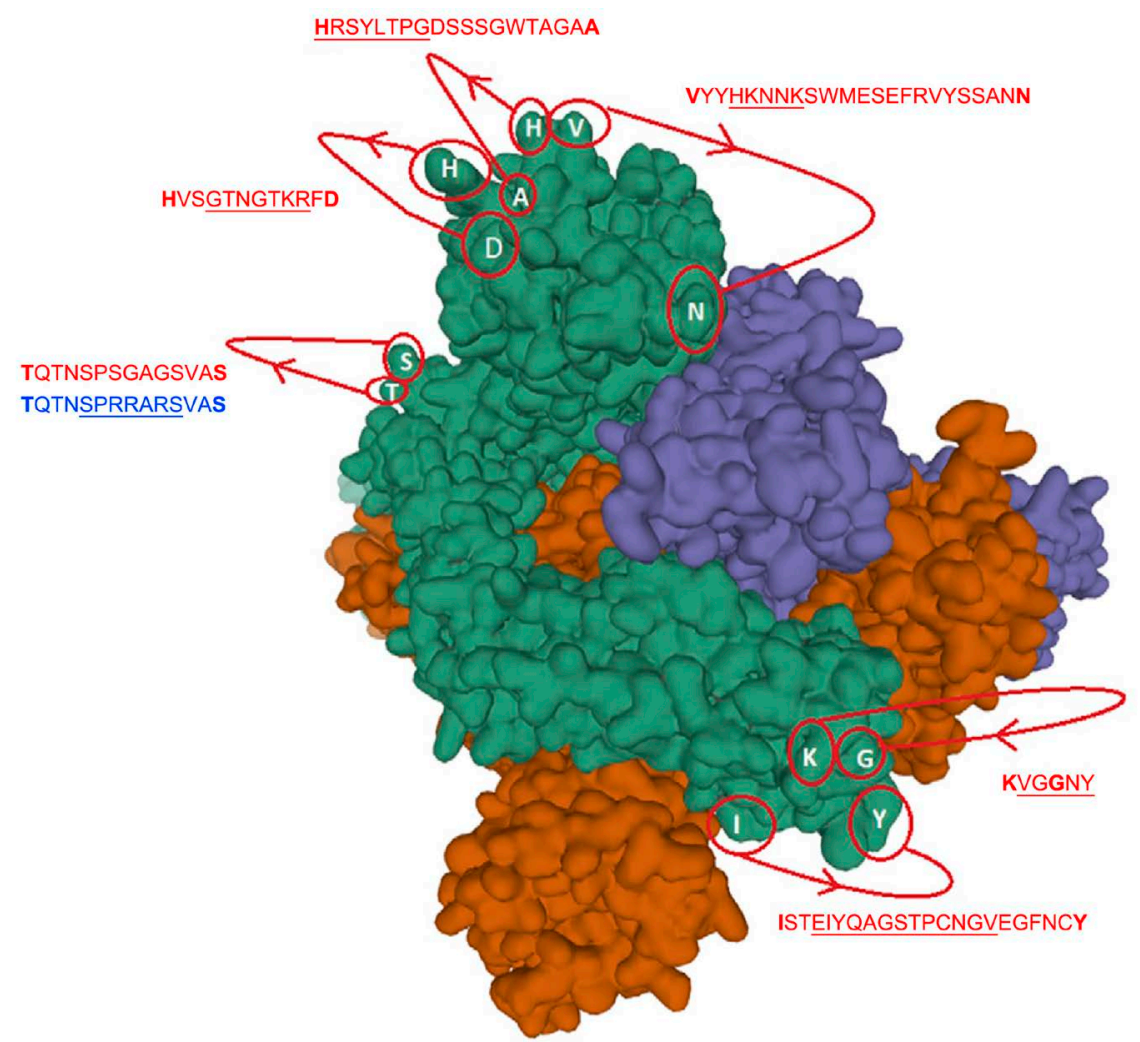

Figure 7. Identified inserts in SARS-CoV-2 examined by electron microscopy [19].

that SARS-CoV-2 was created by a recombination event that occurred inadvertently or consciously in a laboratory handling coronaviruses, with the new virus then accidentally released into the local human population" [21].

As seen previously, geneticists have very powerful tools for genome edition. Using CasX protein, the genetic modification could be invisible. Therefore, the possibility of having SARS-CoV-2 as an unnatural virus (means a manmade Coronavirus) cannot be ignored. A new way of assessing the modification of a gene will be discussed later.

\subsection{Entropy and RNA}

RNA (as well as DNA) is characterized by a sequence of nucleotides. The order and the type of nucleotides, as well as the $3 \mathrm{D}$ structure, are very significant factors on the energetic level that can influence entropy.

The primary structure of RNA is the single strand structure (Figure 8(a)). But RNA strands usually fold back on themselves, forming an intramolecular structure that can be very stable and compact. The basis of this structure is the formation of internal pairings between complementary bases (i.e., Watson-Crick pairing). This is the secondary structure (Figure $8(\mathrm{~b})$ ). This hairpin structure can also fold back on itself and form a 3D structure. This is the tertiary structure (Figure 8(c)).

The stability of the structure depends on the binding energy of the structure. 
(a)

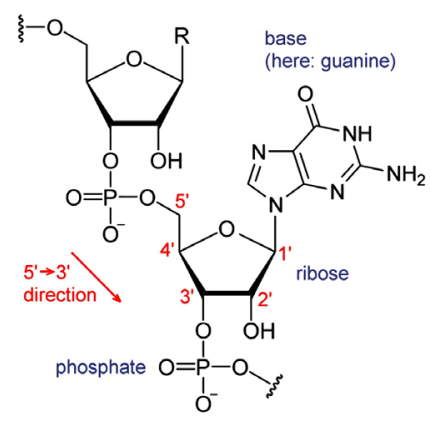

(b)

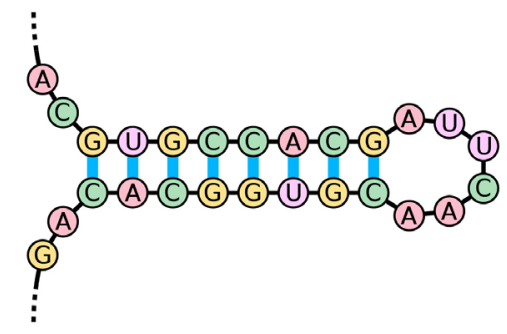

(c)

Figure 8. RNA primary (a) [22], secondary (b) [22] and tertiary (c) [23] structure.

A database detailing the binding energy of nucleotides is existing [24] and used in software (like MFOLD) in order to assess the free energy and the enthalpy of the RNA.

Some studies have shown that the structure plays a key role in an unexpected stability of the RNA. This has been demonstrated as well as for the secondary structure (hairpin structure) [25] but also for the tertiary structure [26]. Usually a natural structure has a lower free-energy level and a low state of entropy. For example, without extra energy, water vapor condenses and therefore reduces its entropy. In the case of RNA, the structure could allow for both a low level of energy (i.e., a very stable structure) but also high entropy (i.e., carrying the force of repulsion).

\section{SARS-CoV-2 Virus on the Subatomic Level}

\subsection{Chemical Modification of Phosphate Used in CRISPR Leads to an Imbalanced State}

In order to improve the cleavage process, genetic engineers have modified the single guide RNA (sgRNA) [27] [28]. In natural RNA and DNA, the phosphate group in the single guide backbone contains an ionized oxygen atom. One of the possible modifications is to replace this ionized oxygen atom by hydroxyl radical [29] (Figure 9).

On a subatomic level, this results in one electron globally missing.

In a previous article, the authors introduced a unifying quantum theory called the I-Theory [1]. The paper described how the I-Theory unifies all major theories and provides a fresh perspective for understanding basic concepts of physics and other branches of science.

In a second article, the specifics concepts of entropy and negentropy were analyzed [2]. In short: 


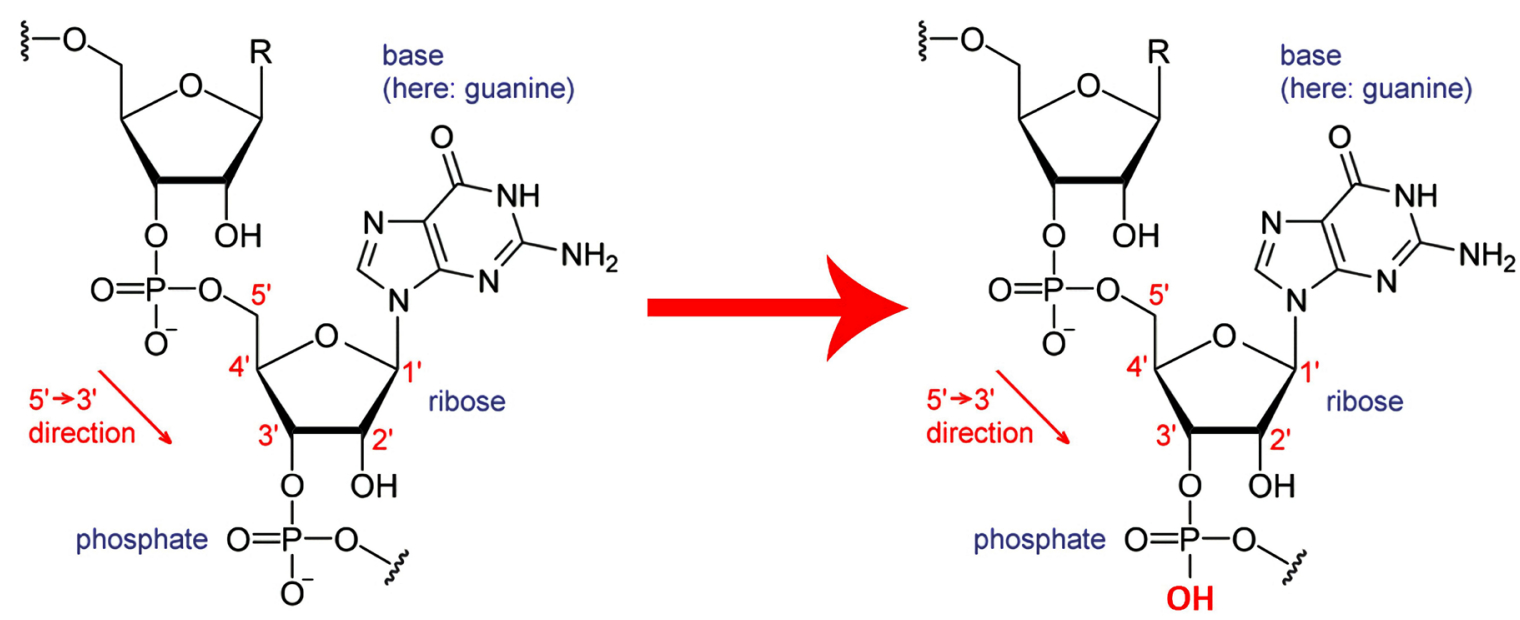

Figure 9. Modification of RNA backbone's phosphate group used in CRISPR.

- If the repelling energy between subatomic building-block particles is reduced, negentropy dominates.

- If the repelling energy between subatomic building-block particles is increased, entropy dominates.

Using the supersymmetry model, the authors [1] gave a new understanding of the particle family (i.e., boson/fermion). They showed that bosons (e.g., photon and gluons) are formed due to the force of repulsion, while fermions (e.g., electrons and quarks) are formed due to the force of attraction.

Therefore, the electron, which is a fermion, that goes missing due to the modification of phosphate group in SARS-CoV-2 leads to an imbalance state between fermions and bosons. The result is a domination of the repulsion force. This extra repulsion force also explains why the CRISPR method can easily create space in the DNA or RNA for the cleavage function.

The missing electrons have also been noticed by Sørensen especially in the binding area (between the S1/S2 proteins) at the precise location of the inserted sequences. He states that there is a high concentration of positive charges on the surface of the receptor binding motif (RBM) (Figure 10) [18].

\subsection{Analysis of the SARS-CoV-2 Virus Case}

In Section 2.4, the possibility that SARS-CoV-2 is not a natural virus is demonstrated. Considering this hypothesis, it needs to be assessed how much it has been modified and how strongly the modification is concentrated on a particular section. Walls [19] and Piplani [20] have shown that the SARS-CoV-2 virus contains at least 6 inserts especially in the spike region, which implies that in this region the repulsion forces are very strong and localized. The electrical charge of inserted sequences plays a key role in the binding of the cell.

Section 2.3 illustrated that using Cas12a or CasX allows for a highly accurate form of genetic modification. Cas12a and CasX act due to numerous weak bonds. A weak bond is characterized by the weak force, or entropy. 


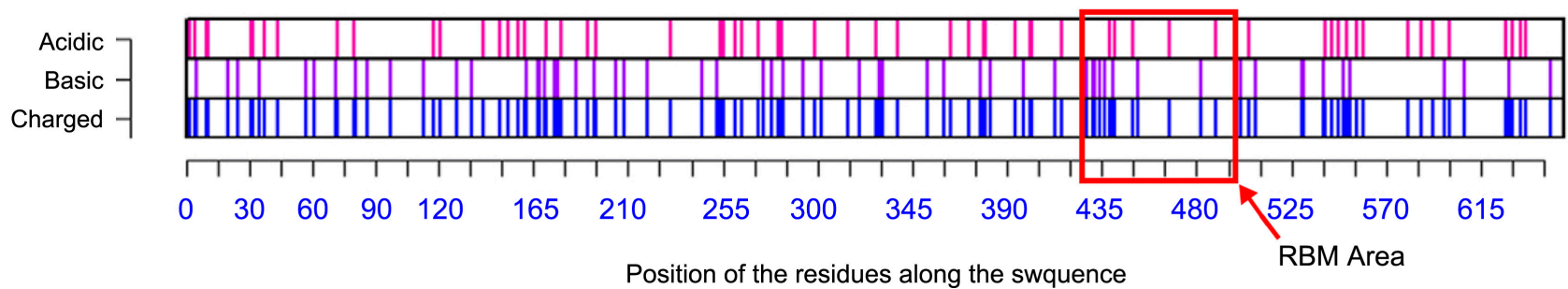

Figure 10. Distribution of charge using a rolling window of 12 amino acids in steps in SARS-CoV-2 [18].

In Section 2.5, it is pointed out that the 3D structure allows for having a stable structure with high entropy.

\subsection{Imbalanced Forces in Infected Cells}

In infected cells, the virus is replicated. Therefore, a large number of SARS-CoV-2 viruses will be in the cell. It was previously shown that the virus contains a very concentrated and powerful repulsion force.

Booth from King's College London has found that some highly affected cells in COVID-19 patients contain no more cytoplasm [30]. Single cells had fused together and some were found to have up to 15 nuclei. These are characteristics of imbalanced forces at the subatomic level, wherein the repulsion force, or entropy, is dominating. Since SARS-CoV-2 virus has a very high level of the force of repulsion (i.e., weak force), the binding is dissolved on the atomic plane, which could certainly weaken cells and destroy the cytoplasm.

It appears that SARS-CoV-2 virus can infect any kind of cell, and thus many organs behave erratically [31] including the lungs, liver, kidneys, blood coagulation, and heart [32]. New symptoms and serious effects are being reported all the time.

\section{Conclusion and Perspectives}

In this paper, the I-Theory and its application to the concept of entropy and negentropy are used in order to a have a deeper understanding of SARS-CoV-2.

It is shown that SARS-CoV-2 is possibly not a natural virus. The modifications in the backbone's phosphate group induced by CRISPR engineering create a highly imbalanced state. The entropy and therefore the repulsion forces strongly dominate in this type of virus.

These imbalanced forces lead to an explosion of the infected cells damaging the organs and creating various and long-term effects.

Since a new generation of vaccines based on mRNA are presently being used, the understanding of how the SARS-CoV-2 virus as well as the vaccine works is of crucial importance, because the laboratories Pfizer and Moderna have declared that genetic engineering was used in the mRNA vaccine development process as well as the mRNA ingredient itself. However, the kinds of modifications which have been done in order to deactivate the SARS-CoV-2 virus are not published yet. More importantly, it is crucial to investigate whether the entro- 
py-negentropy balance still remains, or whether it is reduced or increased. Therefore, coordinated research should be conducted that can answer the following questions thoroughly, before a potential vaccine is released:

1) Which modifications of the mentioned phosphate group occurred in the SARS-CoV-2 virus?

2) How can these modifications be deactivated by a potential vaccine without further disrupting the entropy-negentropy balance?

3) Are there (natural) measures to eliminate the virus before infection?

A new outlook of the SARS-CoV-2 virus based on the I-Theory gives new perspectives to medicine which show and make clear the danger of this virus. With an accurate diagnostic tool, an effective and safe cure to COVID-19 can be found.

This methodology is promising for other areas of medical research too. Stabilizing the entropy-negentropy balance will open up new treatments for other diseases. Cancer, for example, has an excess of the attractive force at the subatomic plane, which leads to tumors. Only when the natural balance between attraction and repulsion is maintained can health be sustained.

\section{Conflicts of Interest}

The authors declare no conflicts of interest regarding the publication of this paper.

\section{References}

[1] Swami Isa, H. and Dumas, C. (2019) I-Theory: A Unifying Theory? Journal of High Energy Physics, Gravitation and Cosmology, 5, 332-359.

[2] Swami Isa, H. and Dumas, C. (2020) Entropy and Negentropy Principles in the I-Theory. Journal of High Energy Physics, Gravitation and Cosmology, 6, 259-273. https://doi.org/10.4236/jhepgc.2020.62020

[3] Seyran, M., Pizzol, D., Adadi, P., et al. (2020) Questions Concerning the Proximal Origin of SARS-CoV-2. Journal of Medical Virology, 93, 1204-1206.

https://doi.org/10.1002/jmv.26478

[4] Peacock, T., Goldhil, D., Zhou, J., et al. (2020) The Furin Cleavage Site of SARS-CoV-2 Spike Protein Is a Key Determinant for Transmission Due to Enhanced Replication in Airway Cells. https://doi.org/10.1101/2020.09.30.318311

[5] Mojica, F., Juez, G. and Rodríguez-Valera, F. (1993) Transcription at Different Salinities of Haloferax Mediterranei Sequences Adjacent to Partially Modified PstI Sites. Molecular Microbiology, 9, 613-621. https://doi.org/10.1111/j.1365-2958.1993.tb01721.x

[6] Barrangou, R., Fremaux, C., Deveau, H., et al. (2007) CRISPR Provides Acquired Resistance against Viruses in Prokaryotes. Science, 315, 1709-1712. https://doi.org/10.1126/science.1138140

[7] Jinek, M., Doudna, J., Charpentier, E., et al. (2012) A Programmable Dual-RNA-Guided DNA Endonuclease in Adaptive Bacterial Immunity. Science, 337, 816-821. https://doi.org/10.1126/science.1225829

[8] Synthego. CRISPR 101. https://www.synthego.com/resources/crispr-101-ebook 
[9] Genethique (2018) Vers une utilisation massive de crispr cas12. https://www.genethique.org/vers-une-utilisation-massive-de-crispr-cas12/

[10] Knott, et al. (2019) Structural Basis for AcrVA4 Inhibition of Specific CRISPR-Cas12a. eLife. https://doi.org/10.7554/eLife.49110.037

[11] Koonin, et al. (2017) Diversity, Classification and Evolution of CRISPR-Cas Systems. Current Opinion in Microbiology, 37, 67-78.

https://doi.org/10.1016/j.mib.2017.05.008

[12] Gasiunas, et al. (2012) Cas9-crRNA Ribonucleoprotein Complex Mediates Specific. PNAS, 109, E2579-E2586. https://doi.org/10.1073/pnas.1208507109

[13] Cofsky, et al. (2020) CRISPR-Cas12a Exploits R-Loop Asymmetry to Form Double-Strand Breaks. eLife, 9, e55143. https://doi.org/10.7554/eLife.55143

[14] Genethique (2019) CRISPR CasX, un ciseau moléculaire concurrent de CRISPR Cas9.

https://www.genethique.org/crispr-casx-un-ciseau-moleculaire-concurrent-de-crisp $\underline{\mathrm{r} \text {-cas } 9}$

[15] Liu, et al. (2019) CRISPR-CasX Is an RNA-Dominated Enzyme Active for Human Genome Editing. Nature, 566, 218-223. https://doi.org/10.1038/s41586-019-0908-x

[16] The_Sun (2020) PROVE New COVID Strain Is 70\% More Contagious-It's Just Causing Panic and Fear, Experts Urge.

https://www.thesun.co.uk/news/13535213/prove-new-covid-strain-70-more-contagi ous-experts-urge

[17] Carfi, A., et al. (2020) Persistent Symptoms in Patients after Acute COVID-19. $J A M A, 324,603-605$. https://doi.org/10.1001/jama.2020.12603

[18] Sørensen, B., Susrud, A. and Dalgleish, A. (2020) Biovacc-19: A Candidate Vaccine for Covid-19 (SARS-CoV-2) Developed from Analysis of its General Method of Action for Infectivity. QRB Discovery-Cambridge Coronavirus Collection. https://doi.org/10.1017/qrd.2020.8

[19] Walls, A. (2020) Structure, Function, and Antigenicity of the SARS-CoV-2 Spike Glycoprotein. Cell, 181, 281-292. https://doi.org/10.1016/j.cell.2020.02.058

[20] Piplani, S., Singh, P. and Winkler, D. (2020) In Silico Comparison of Spike Protein-ACE2 Binding Affinities across Species; Significance for the Possible Origin of the SARS-CoV-2 Virus. https://arxiv.org/abs/2005.06199

[21] White, N. (2020) No Known Animal Host and "Almost Perfect" Human Adaption: Top Australian Vaccine Scientist Reveals How COVID-19's Unique Structure Means It's Either Man-Made or a "Complete Fluke" of Nature.

https://www.dailymail.co.uk/news/article-8356751/How-COVID-19s-unique-struct ure-means-man-made.html

[22] Wikipedia. Acide ribonucléique. https://fr.wikipedia.org/wiki/Acide_ribonucl\%C3\%A9ique

[23] PDB-101. Transfer RNA. http://pdb101.rcsb.org/motm/15

[24] Freier, S., et al. (1986) Improved Free-Energy Parameters for Predictions of RNA. Biochemestry, 83, 9373-9377. https://doi.org/10.1073/pnas.83.24.9373

[25] Tuerk, C., et al. (1988) CUUCGG Hairpins: Extraordinarily Stable RNA Secondary Structures Associated with Various Biochemical Processes. Proceedings of the National Academy of Sciences of the United States of America, 85, 1364-1368.

https://doi.org/10.1073/pnas.85.5.1364

[26] Aalberts, P. (2011) Loop Entropy Assists Tertiary Order: Loopy Stabilization of 
Stacking Motifs. Entropy, 13, 1958-1966. https://doi.org/10.3390/e13111958

[27] Yin, H., Song, C.-Q., Suresh, S., et al. (2017) Structure-Guided Chemical Modification of Guide RNA Enables Potent Non-Viral in Vivo Genome Editing. Nature Biotechnology, 35, 1177-1188.

[28] Ryan, D., et al. (2018) Improving CRISPR-Cas Specificity with Chemical Modifications in Single-Guide RNAs. Nucleic Acids Research, 46, 792-803.

https://doi.org/10.1093/nar/gkx1199

[29] Filippova, J., et al. (2019) Guide RNA Modification as a Way to Improve CRISPR/ Cas9-Based Genome-Editing Systems. Biochimie, 167, 49-60.

https://doi.org/10.1016/j.biochi.2019.09.003

[30] Booth, R. (2020) Covid-19 Can Damage Lungs of Victims beyond Recognition, Expert Says.

https://www.theguardian.com/world/2020/jun/15/covid-19-can-damage-lungs-victi ms-beyond-recognition-expert-says

[31] Zhu, Y.J., et al. (2020) Evaluation of Organ Function in Patients with Severe COVID-19 Infections. Medicina Clinica, 155, 191-196.

https://doi.org/10.1016/j.medcle.2020.05.015

[32] Kolata, G. (2020) A Heart Attack? No, It Was the Coronavirus. https://www.nytimes.com/2020/03/27/health/coronavirus-cardiac-heart-attacks.htm $\underline{1}$ 